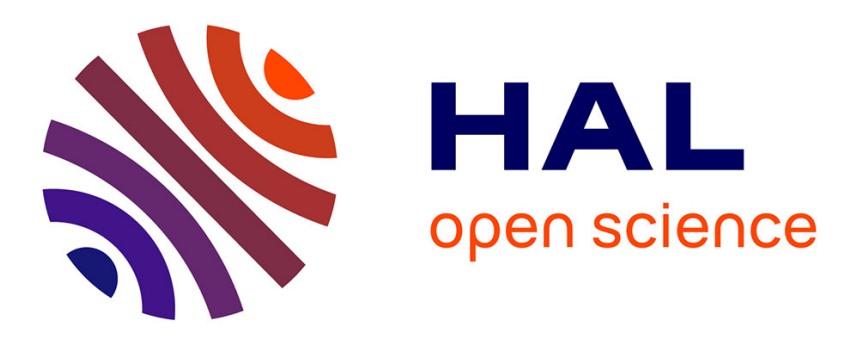

\title{
An S-band Ultrawideband Time Reversal-based RADAR for Imaging in Cluttered Media
}

\author{
Lucio Bellomo, Sebastien Pioch, Marc Saillard, E. Spano
}

\section{To cite this version:}

Lucio Bellomo, Sebastien Pioch, Marc Saillard, E. Spano. An S-band Ultrawideband Time Reversalbased RADAR for Imaging in Cluttered Media. IEEE International Symposium on Phased Array Systems and Technology (ARRAY), 2010, Oct 2010, Boston, United States. pp.394-397. hal-00703411

\section{HAL Id: hal-00703411 \\ https://hal.science/hal-00703411}

Submitted on 1 Jun 2012

HAL is a multi-disciplinary open access archive for the deposit and dissemination of scientific research documents, whether they are published or not. The documents may come from teaching and research institutions in France or abroad, or from public or private research centers.
L'archive ouverte pluridisciplinaire HAL, est destinée au dépôt et à la diffusion de documents scientifiques de niveau recherche, publiés ou non, émanant des établissements d'enseignement et de recherche français ou étrangers, des laboratoires publics ou privés. 


\title{
An S-band Ultrawideband Time Reversal-based RADAR for Imaging in Cluttered Media
}

\author{
L. Bellomo, S. Pioch, M. Saillard and E. Spano \\ LSEET, UMR CNRS 6017, Université du Sud Toulon-Var, France, \{bellomo,pioch,saillard,spano\} @1seet.univ-tln.fr
}

\begin{abstract}
This work presents a new RADAR prototype built for the purpose of imaging targets located in a cluttered environment. The system is capable of performing Phase Conjugation experiments in the ultrawideband [2-4] $\mathrm{GHz}$. In addition, applying the D.O.R.T. method to the inter-element matrix allows us to selectively focus onto targets, hence reducing the clutter contribution. The system has been validated by phsyically backpropagating the focusing wave into the medium all over the frequency band and observing the expected focusing properties.
\end{abstract}

\section{INTRODUCTION}

The concept of Time Reversal Mirror [1] has generated numerous studies in both Acoustics and Electromagnetism in the last decade. Many are the potential applications in nondestructive control, medical imaging, sub-marine acoustics, telecommunications and RADAR. The capacity of these mirrors to focus onto an object is very useful for imaging in random media as it permits to improve the signal-to-clutter ratio and to increase the robustness of the imaging algorithms. In [2] such properties have been demonstrated with synthetic data by including the response to the focusing wave in the solution of the non-linear inverse scattering problem. Our aim here is to build a prototype that allows us to get the data required by this inversion algorithm and to evaluate its performances.

In Electromagnetism, only a few experiments of Time Reversal (TR) have been performed. A first set [3] has been achieved directly in the time domain with a Digital Oscilloscope and an Arbitrary Waveform Generator (AWG). In a second kind of experiment $[4,5]$, the system works in the frequency domain with a Vector Network Analyzer (VNA). In the first approach the experimental backpropagation of the focusing wave is more easily performed thanks to the AWG, whereas in the second one the re-transmission is only done numerically and one ought to rather talk about Phase Conjugation over a given bandwidth and pulse synthesis. Yet the available bandwidths are smaller in the former case despite the recent progress of commercial AWG's.

Our prototype merges the advantages of both described solutions. It is made of a linear 8-antenna array, working in the frequency domain and capable of experimentally re-transmit the complex conjugate of the received signal within a band- width of $2 \mathrm{GHz}$ at S-band. It also allows us to apply the D.O.R.T. (french acronym for Décomposition de l'Opérateur de Retournement Temporel) method [6], which is of particular interest to focus selectively onto a target. In case of multiple targets, it is in fact more powerful than Time Reversal, which has to be iterated and which restricts focusing to the brightest target.

We present here the prototype and the first experimental results conducted to validate it. They consist of an UWB Phase Conjugation experiment, equivalent after IFFT to Time Reversal, where an additional antenna is the source onto which we want to focalize the time-reversed wave, and of a D.O.R.T. experiment where a passive target is detected and illuminated. These results encourage us to explore the possiblity of using these methods for quantitave imaging of targets in cluttered configurations.

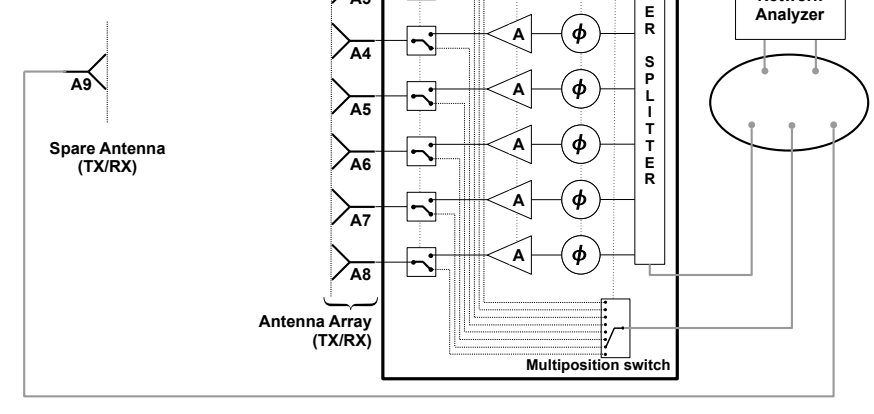

Figure 1 - Prototype architecture.

\section{RADAR DESCRIPTION}

The architecture of our RADAR (Fig. 1) is built around a 2port VNA serving both as signal source and receiver. The RF front-end is made of a linear array of 8 UWB antennas (A1-A8) plus one more spare antenna (A9), working in a multistatic configuration. Antennas are antipodal symmetric Exponentially Tapered Slot Antennas (ETSA) [7]; they 


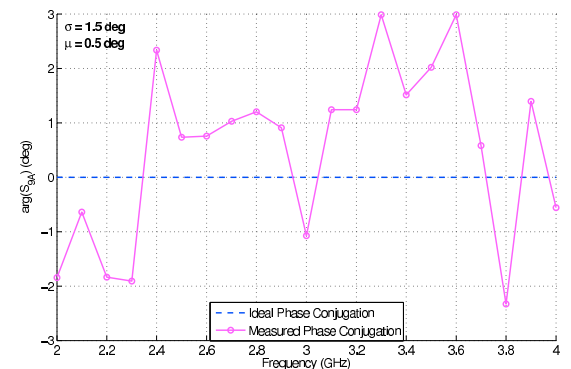

(a)

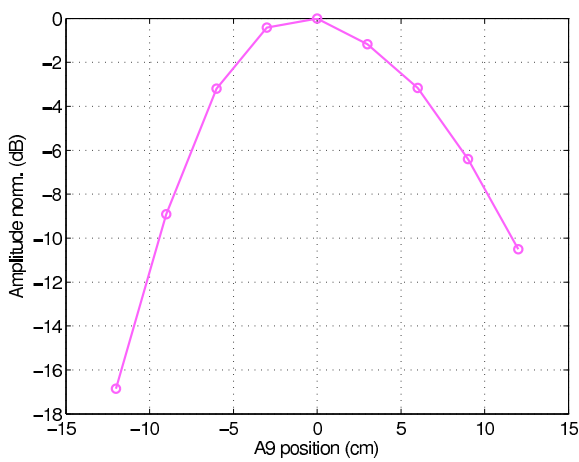

(b)

Figure 2 - UWB Phase Conjugation experiment. (a) Phase of the ideal (dashed blue) and measured (magenta) backpropagated signal versus frequency and (b) largest amplitude value of the synthesized backpropagated pulse as a function of the position of A9 with respect to the initial location.

show a very good input impedance matching $(\mathrm{SWR}<2)$ in the [2-18] GHz frequency band and radiate a vertically-polarized (direction perpendicular to the plane of Fig. 1) electric field. Each array channel is controlled both in amplitude and phase via wideband attenuator/phase shifter $(\mathrm{A} / \Phi)$ couples driven numerically. We also dispose of an eight-switch bank connected to the antennas and of a an additional multiposition switch. Finally, a power splitter allows to recombine/split the eight channels. Antennas and phase shifters respectively impose the low and high boundaries of the exploitable [24] $\mathrm{GHz}$ frequency band.

The RF section is controlled through a PIC microcontroller connected via USB to the PC. All measurements are carried out in a $1.5 \mathrm{~m} \times 0.6 \mathrm{~m}$ chamber made of flat absorbing panels.

\section{VALIDATING EXPERIMENTS AND RESULTS}

\section{UWB Phase Conjugation experiment}

The setup of the experiment is the following. A9 is the source antenna and is placed in front of the array antenna $A 1,35 \mathrm{~cm}$ away. The field radiated by A9 is initially recorded by each antenna of the array. Since our system works in the frequency

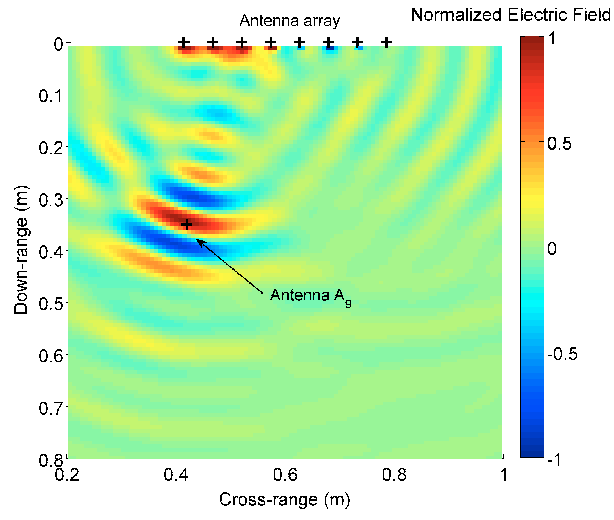

Figure 3 - UWB Phase Conjugation experiment. Timedomain field chart at the instant when the re-emitted wave focuses on A9.

domain, Time Reversal is equivalent by Fourier Transform properties to a Phase Conjugation of the received wave [1]. Hence this recorded vector is conjugated at each frequency and backpropagated by coding the amplitude and phase law into the $\mathrm{A} / \Phi$ couples. Finally, A9 measures the field re-transmitted by the array. Because of the reciprocity of the system and the "peculiar" array phase law, the phase of the signal measured by A9 must be equal to zero at each frequency. Confirmating this, in Fig. 2(a) we have recorded a mean phase of 0.5 deg with standard deviation of $1.5 \mathrm{deg}$ over the whole bandwidth. In addition, when moving A9 away from its original position the backpropagated wave amplitude must be smaller, since there is no reason for a constructive interference phenomenon to happen at a different location. Such spatial focusing property is indeed shown in Fig. 2(b), where the decrease in the largest value of the synthesized backpropagated pulse for different A9 positions is clear.

A final analysis of the experiment consists in a more classical numerical backpropagation of the conjugated received wave over the whole frequency range. To do this we simply model our antennas as infinitesimal dipoles of vertical moment in free-space. This modeling choice introduces an error in the Time Reversal process because it adds a mismatch between the characteristics of the antennas in the experimental data and in the synthetic backpropagation, invalidating the reciprocity theorem. Nevertheless, it proves to be rather effective especially because our antennas have a very stable phase center over the [24] $\mathrm{GHz}$ bandwidth, exactly as infinitesimal dipoles do. For each frequency we produce a field chart, showing the map of the amplitude of the electric field inside our chamber. Finally, by taking the IFFT of the field values at each pixel, we generate a field chart for every time instant, which allows us to observe the focusing process in the timedomain. Such a chart at the instant where the focusing spot is best concentrated around the position of A9 is presented in Fig. 3. 


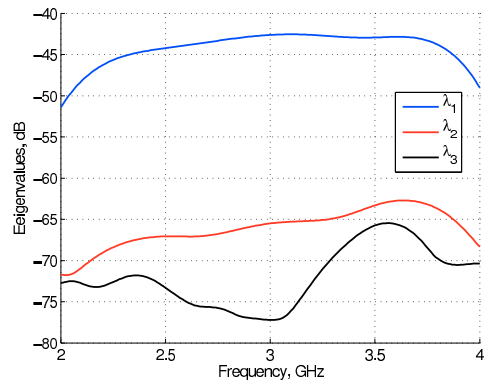

(a)

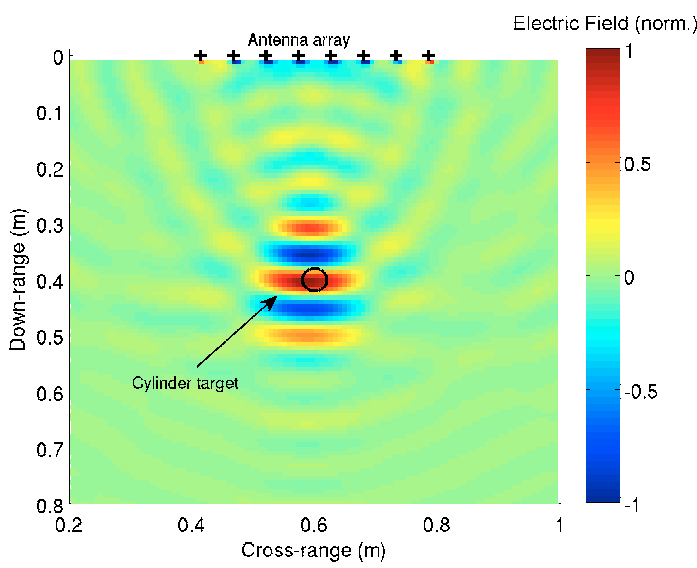

(b)

Figure 4 - UWB D.O.R.T. experiment. (a) Array interelement matrix eigenvalues distribution versus frequency and (b) one frame of the synthetic time-domain field chart movie associated to the largest eigenvalue.

\section{D.O.R.T. experiment}

The second experiment is an application of the D.O.R.T. method. In the experimental setup A9 is removed and a $4 \mathrm{~cm}$-diameter metallic cylinder located $40 \mathrm{~cm}$ away from the array center is used as diffracting object. Since the array antennas act both as sources and receivers, an inter-element $8 \times 8$ matrix is measured at each frequency $\omega$; the difference between two such matrices recorded with and without the scatterer in place is finally used in order to reduce antenna coupling effects [5]. The D.O.R.T. method is based on the Singular Value Decomposition of this matrix, the transfer matrix $\mathbf{K}(\omega)$, which writes as $\mathbf{U}(\omega) \boldsymbol{\Lambda}(\omega) \mathbf{V}^{H}(\omega)$, where ${ }^{H}$ stands for conjugate-transpose. The diagonal elements of $\boldsymbol{\Lambda}(\omega)$ are the singular values, whose hierarchy provides information about the brightness of the targets, and the columns of $\mathbf{V}(\omega)$ are the associated right singular vectors, whose complex amplitudes are to be fed to the array to focus selectively onto each target without any iteration.

The behavior of the three largest singular values against frequency is presented in Fig. 4(a); the largest one, $\lambda_{1}(\omega)$, corresponds to the target, while the associated singular vector $\mathbf{v}_{1}(\omega)$ supplies the amplitude and phase law needed to generate a wave focusing onto it. As in the previous experiment, we
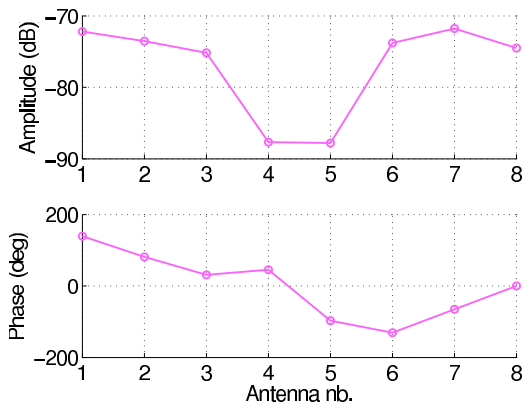

(a)

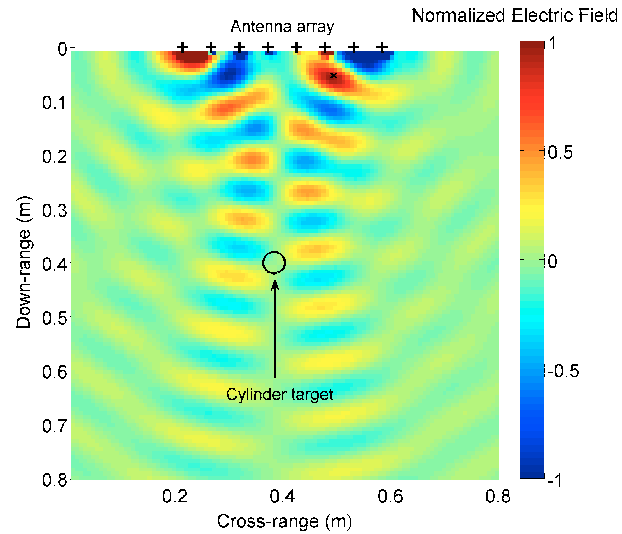

(b)

Figure 5 - UWB D.O.R.T. experiment. Anti-symmetric singular vector (associated to $\lambda_{2}$ in Fig. 4) (a) complex law and (b) real part of the corresponding monochromatic field chart at $3 \mathrm{GHz}$.

have coded such vector into the system at each frequency and we have measured the object response to the focusing wave.

Such response will be used to enhance the performances of inversion algorithms as shown with synthetic data in [2]. One can also simulate the backpropagation of this wave and build a chart of the field distribution over the area of interest. As in the previous experiment, the antennas are simply modeled as vertical electric dipoles. The result of this processing in the time-domain is a movie showing the focusing wave propagating towards the target. The frame at the instant when it converges onto the cylinder is given in Fig. 4(b), where one can appreciate the focusing of the wave onto the target. The cross-range resolution (half of the focusing region along the direction parallel to the array) is $\approx 8 \mathrm{~cm}$, in accordance with the theoretical limit $\lambda F / D$, where $F$ is the distance between the array and the target $(40 \mathrm{~cm}), D$ is the array aperture $(35 \mathrm{~cm})$ and $\lambda$ is here the wavelength at the highest frequency $4 \mathrm{GHz}(7.5 \mathrm{~cm})[8]$.

The second largest singular value, $\lambda_{2}(\omega)$ is also related to the target. In effect, it has been shown in [9] that for a linear array of antennas the singular values hierarchy is intimately linked to the decomposition of the diffracted field into a Bessel-Fourier series along the azimuth angle $\theta$. In particular, $\lambda_{2}(\omega)$ is associated to the first anti-symmetric field com- 
ponent, which has zero amplitude around the center of the array and opposite phases in the two halves. This is shown in Fig. 5(a), showing the amplitude and phase of the components of $\mathbf{v}_{2}$ for $\omega=3 \mathrm{GHz}$. Indeed, the amplitude decreases of around $10 \mathrm{~dB}$ and the phase has a jump of nearly $180^{\circ}$ around the middle antennas. The real part of the corresponding monochromatic field chart is shown in Fig. 5(b) confirms this, with a zero at the position of the target and opposite phases in the two half-planes corresponding to the two halves of the array.

The lower singular values in Fig. 4(a) are not exploitable anymore due to the limited dynamic range of the system; this can be noticed by the fact that $\lambda_{3}(\omega)$ is noisy with respect to $\lambda_{1}(\omega)$ and $\lambda_{2}(\omega)$, which run almost parallel along the system bandwidth.

\section{Conclusions}

We have manufactured a Time Reversal RADAR prototype working in the [2-4] GHz frequency band. The validation of the system has been achieved through two experiments, the second of which represents to our best knowledge the first experimental report of electromagnetic D.O.R.T. singular vector re-emission over such a wide frequency band. This setup will allow us to gather experimental data for testing the use of Time Reversal-based methods in combination with inversion algorithms.

\section{ACKNOWLEDGEMENTS}

This work is financed by DGA/CNRS and by THALES AIR SYSTEMS S.A. through a collaboration contract. The ETSA antennas have been kindly offered by LEAT in Nice-Sophia Antipolis, France.

\section{REFERENCES}

[1] M. Fink, C. Prada, F. Wu, and D. Cassereau, "Self focusing in inhomogeneous media with time reversal acoustic mirrors," in Proc. IEEE Ultrasonics Symposium, vol. 2, Montreal, Que., Canada, Oct. 1989, pp. 681-686.

[2] A. Dubois, K. Belkebir, and M. Saillard, "Localization and characterization of two-dimensional targets buried in a cluttered environment," Inv. Probl., vol. 20, no. 6, pp. S63-S79, Nov. 2004.

[3] G. Lerosey, J. de Rosny, A. Tourin, A. Derode, and M. Fink, "Time reversal of wideband microwaves," Applied Physics Letters, vol. 88, no. 15, p. 154101, Apr. 2006.
[4] D. Liu, S. Vasudevan, J. Krolik, G. Bal, and L. Carin, "Electromagnetic Time-Reversal Source Localization in Changing Media: Experiment and Analysis," IEEE Trans. Antennas Propag., vol. 55, no. 2, pp. 344-354, Feb. 2007.

[5] A. Cresp, I. Aliferis, M. J. Yedlin, J.-Y. Dauvignac, and C. Pichot, "Time-domain processing of electromagnetic data for multiple-target detection," AIP Conf. Proc., 3rd Conference on Mathematical Modeling of Wave Phenomena, vol. 1106, no. 1, pp. 204-213, Mar. 2009.

[6] C. Prada and M. Fink, "Eigenmodes of the time reversal operator: A solution to selective focusing in multipletarget media," Wave Motion, vol. 20, pp. 151-163, Sep. 1994.

[7] E. Guillanton, J. Y. Dauvignac, C. Pichot, and J. Cashman, "A new design tapered slot antenna for ultrawideband applications," Microwave and Optical Technology Letters, vol. 19, no. 4, pp. 286-289, 1998.

[8] M. Born and E. Wolf, Principles of Optics, 1970.

[9] G. Micolau and M. Saillard, "D.O.R.T. method as applied to electromagnetic subsurface sensing," Radio Sci., vol. 38, no. 3, pp. S63-S79, May 2003. 